\title{
Letter
}

\section{Post Post-Broadcast Democracy? News Exposure in the Age of Online Intermediaries}

\author{
SEBASTIAN STIER GESIS-Leibniz Institute for the Social Sciences, Germany
}

FRANK MANGOLD University of Hohenheim, Germany

MICHAEL SCHARKOW Johannes Gutenberg University Mainz, Germany

JOHANNES BREUER GESIS-Leibniz Institute for the Social Sciences, Germany, and Center

for Advanced Internet Studies, Germany

\begin{abstract}
$O$ nline intermediaries such as social network sites or search engines are playing an increasingly central role in democracy by acting as mediators between information producers and citizens. Academic and public commentators have raised persistent concerns that algorithmic recommender systems would negatively affect the provision of political information by tailoring content to the predispositions and entertainment preferences of users. At the same time, recent research indicates that intermediaries foster exposure to news that people would not use as part of their regular media diets. This study investigates these unresolved questions by combining the web browsing histories and survey responses of more than 7,000 participants from six major democracies. The analysis shows that despite generally low levels of news use, using online intermediaries fosters exposure to nonpolitical and political news across countries and personal characteristics. The findings have implications for scholarly and public debates on the challenges that high-choice digital media environments pose to democracy
\end{abstract}

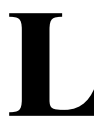
iberal democracy is facing threats around the globe. In many academic and public accounts of the current perils of democracy, the high-choice media environment brought about by the Internet is prominently mentioned among the culprits. While mass broadcasters and newspapers have traditionally guaranteed common exposure to public-affairs information, the ever-expanding variety of digital entertainment and niche content has provided citizens with unprecedented autonomy in their information choices (Van Aelst et al. 2017). The algorithmically and socially driven recommender systems of online intermediaries such as search engines (e.g., Google), social network sites (e.g.,

Sebastian Stier (D), Senior Researcher, Department Computational Social Science, GESIS - Leibniz Institute for the Social Sciences, Germany, sebastian.stier@gesis.org.

Frank Mangold (D), Postdoctoral Researcher, Department of Media Research and Media Use, University of Hohenheim, Germany, frank.mangold@uni-hohenheim.de.

Michael Scharkow, Professor, Department of Communication, Johannes Gutenberg University, Mainz, Germany, scharkow@unimainz.de.

Johannes Breuer (D), Senior Researcher, Department Survey Data Curation, GESIS - Leibniz Institute for the Social Sciences, Germany, and Team Leader, Team Research Data and Methods, Center for Advanced Internet Studies (CAIS), Germany, johannes. breuer@gesis.org.

Received: December 17, 2020; revised: June 29, 2021; accepted: September 20, 2021. First published online: October 29, 2021.
Facebook), or online portals (e.g., MSN) potentially even amplify the scope of audience self-selection. Therefore, intermediaries seem to be a natural continuation of the "post-broadcast democracy" of the 1990s and 2000s that was characterized by selective avoidance of public-affairs coverage in favor of entertainment content (Prior 2007). Yet not least due to the limited accuracy of self-reported media use (Prior 2009), studying the dynamics of news exposure in digital media environments remains a major challenge.

Against this backdrop, we ask, do online intermediaries indeed drive citizens away from news? Or do they actually foster - nonpolitical and political - news exposure? To this end, we combine cross-national data on observed web browsing behavior with the complementary advantages of surveys and content analysis (Stier et al. 2020). The within-person regression models show that even across countries and personal characteristics, intermediaries foster rather than restrict news exposure. These findings are relevant for the fields of political communication and public opinion but also have broader implications for media policy and democracy.

\section{ONLINE INTERMEDIARIES AND EXPOSURE TO NEWS}

The emergence of intermediaries as central brokers between information producers and citizens in digital 
media environments marks a significant evolutionary stage in media development (Van Aelst et al. 2017). Recent scholarship has gravitated between two theoretical poles when it comes to intermediaries' effects on news use.

One school has argued that intermediaries exacerbate the trend toward individualized media diets and selective avoidance of public-affairs coverage that has accompanied the expansion of television broadcasting (Prior 2007). It was expected that users maintain their regular selection patterns when allocating their attention to social media content (Kümpel 2020; Van Aelst et al. 2017). In fact, these predispositions should be amplified due to curated content flows on intermediaries such that search and recommender systems may show more news to politically interested citizens in the first place (Thorson 2020). Proponents of concepts such as "echo chambers" or "filter bubbles" even anticipate that the most engaged citizens end up being trapped in enclaves of like-minded speech (Sunstein 2009). However, in light of a growing body of evidence showing that ideological self-selection is not widespread (Barberá 2015; Flaxman, Goel, and Rao 2016; Fletcher, Robertson, and Nielsen 2021; Guess 2021; Tucker et al. 2018), the more severe consequence would be that people tune out or get tuned out of news altogether (Fletcher, Robertson, and Nielsen 2021; Skovsgaard and Andersen 2020; Thorson 2020; Van Aelst et al. 2017).

Another stream of research contends that intermediaries counteract selective exposure by guiding people to news that they would not use as part of their regular daily online routines and content choices (Fletcher and Nielsen 2018a; Möller et al. 2020; Scharkow et al. 2020). Although people may go online for using other types of contents or services, they may encounter news on search engines (Fletcher and Nielsen 2018b), while logging into email accounts on online portals or via social network sites, where popularity cues alongside traditional source cues determine the perceived relevance of contents (Anspach 2017; Bode 2016). As online social network ties stem from heterogeneous domains of life (e.g., work, family, or childhood friends), they have the potential to foster exposure to political content beyond citizens' own interests and viewpoints (Barberá 2015). Although mere encounters of news previews and headlines on intermediary platforms are arguably of restricted democratic value, if citizens are led to news outlets and engage with the full story, this bears potential for gains in political knowledge traditionally attributed to newspaper use (Lee and Kim 2017). Less obvious to users, the inherently social nature of digital media also extends to search engines and portals whose algorithms showcase trending topics from popular news sources that have generated user attention beyond specialized niche audiences.

Using a novel cross-country dataset, this study addresses several gaps in the literature. While some research has advanced that intermediaries amplify inequalities in news use, at least among the politically least engaged (Kümpel 2020; Skovsgaard and Andersen 2020), other studies have identified reverse patterns (Bode 2016; Fletcher and Nielsen 2018a).
Methodologically, survey studies have suffered from the well-known limitations of self-reports of media use (Prior 2009) that are particularly severe when people arrive at online news via intermediaries (Kalogeropoulos, Fletcher, and Nielsen 2019). Experimental studies simulating online environments have only limited external validity, and much tracking research has rested on highly aggregated behavioral data, making it impossible to determine individual news diets (Stier et al. 2020). Apart from some cross-national research (Barberá 2015; Fletcher and Nielsen 2018a), prior findings were confined to isolated cases, most often in the United States. Finally, in light of normative theories of a democratic public sphere, it is crucial to assess the externalities of online intermediaries across different strata of the population and whether positive effects extend to political content.

\section{RESEARCH DESIGN}

To mitigate the deficits of self-reports of media exposure, this study relies on recordings of individual web browsing histories. The "web tracking" data were collected in six Western democracies: France, Germany, Italy, Spain, the United Kingdom, and the United States. By including Democratic Corporatist, Polarized Pluralist, and Liberal media systems, the sample captures well-established differences in political parallelism, journalistic professionalism, and regulatory models (Hallin and Mancini 2004). The case selection also covers two-party and multiparty systems as well as different civic cultures. In addition, the relevance of online intermediaries for getting news differs across the six countries (see supplementary materials [SM] Table S2). If effects of using online intermediaries are consistent in this country sample, it is likely that the findings apply to developed democracies in general.

We recruited 7,775 study participants from the participant pool of the market research company Netquest that maintains online access panels with a continuous web tracking. Participants had given their informed consent and received incentives to install tracking tools and keep them active on their desktop computers. ${ }^{1}$ In total, the data comprises 136 million website visits from March 15 to June 16, 2019. Our surveys of the same study participants covered important variables that have been identified as sources of inequality in news exposure. Descriptive statistics of the sample composition and all used variables can be found in SM S1 and S4. ${ }^{2}$ Despite the nonprobabilistic sampling of participants, the data enable a detailed investigation of the effects of intermediary use on news exposure. Furthermore, online and offline news exposure and privacy

\footnotetext{
${ }^{1}$ Participants could pause the tracking tool at any time. The median number of active days is 71 out of a maximum of 94

${ }^{2}$ Replication materials are available at the American Political Science Review Dataverse (Stier et al. 2021). The raw web tracking data and textual content of website URLs cannot be shared due to proprietary restrictions and to protect the privacy of the study participants.
} 
attitudes of study participants resemble the patterns in external benchmarks (SM S5).

For identifying relevant website visits, the top 5,000 visited domains and most used intermediary platforms in each country were hand coded, covering $89 \%$ of all website visits (see SM S3 for a description of the coding and summary statistics). The identified 556 news domains were further grouped into seven news types like public broadcasting or hyperpartisan news, with different styles of coverage. To construct a measure of political news exposure, we crawled the texts of all visited news URLs and trained a machinelearning classifier (see SM S6 for a description and evaluation). We applied a visit threshold of 10 seconds to establish that respondents had at least somewhat engaged with a website (Lee and Kim 2017; Scharkow et al. 2020), after merging subsequent visits of the same URL to account for automatically reloading browser tabs.

\section{RESULTS}

To establish an empirical baseline, we ran a logistic regression model with person-level random intercepts that account for between-person differences in overall online activity. The model yielded that news accounted for only a small proportion of online activity, with estimated baseline probabilities of a news visit ranging from 0.005 for US participants to 0.0157 for Spanish participants (Figure S6, descriptive statistics are in SM S4). To test a central tenet of our theory, Figure 1 breaks down the probability of a news visit conditional on the previously visited website. Despite differences in the base levels of news exposure, there were substantively large effects of using intermediaries across all countries. Compared with visits of other websites or direct visits (the first visit of a browsing session), being on portals, Facebook, search engines, or Twitter increased the likelihood that the next URL is a news domain. Visits of news domains were also more likely to be followed by a different news domain, whereas the e-commerce platform eBay - which was included as a placebo test-had the weakest effect on subsequent news exposure. However, this clickstream analysis ignores indirect pathways from intermediaries to news (e.g., being primed by news-related content but visiting news sites later) and also does not encapsulate whether intermediaries foster news exposure beyond online users' regular media diets.

FIGURE 1. Probability of News Exposure, Conditional on the Previous Visit

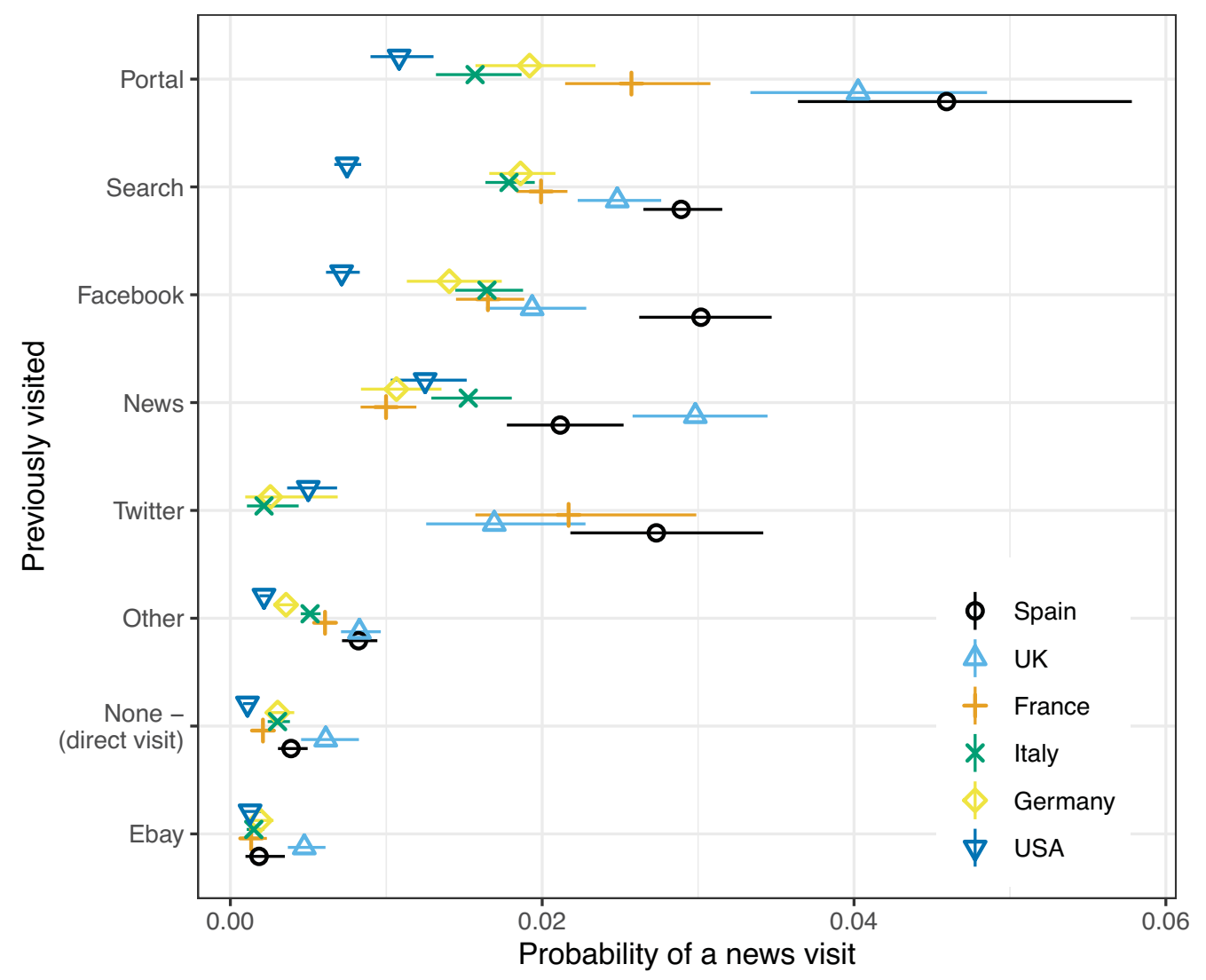

Note: Estimated marginal probabilities and $99 \%$ confidence intervals from a logistic regression model with person-level random intercepts. $N=27,028,342$ domain visits. Subsequent URLs of the same domain were merged. Figure S7 shows the model results for political news visits. 


\section{FIGURE 2. Within-Person Effects of Daily Intermediary Use on Daily News Exposure}

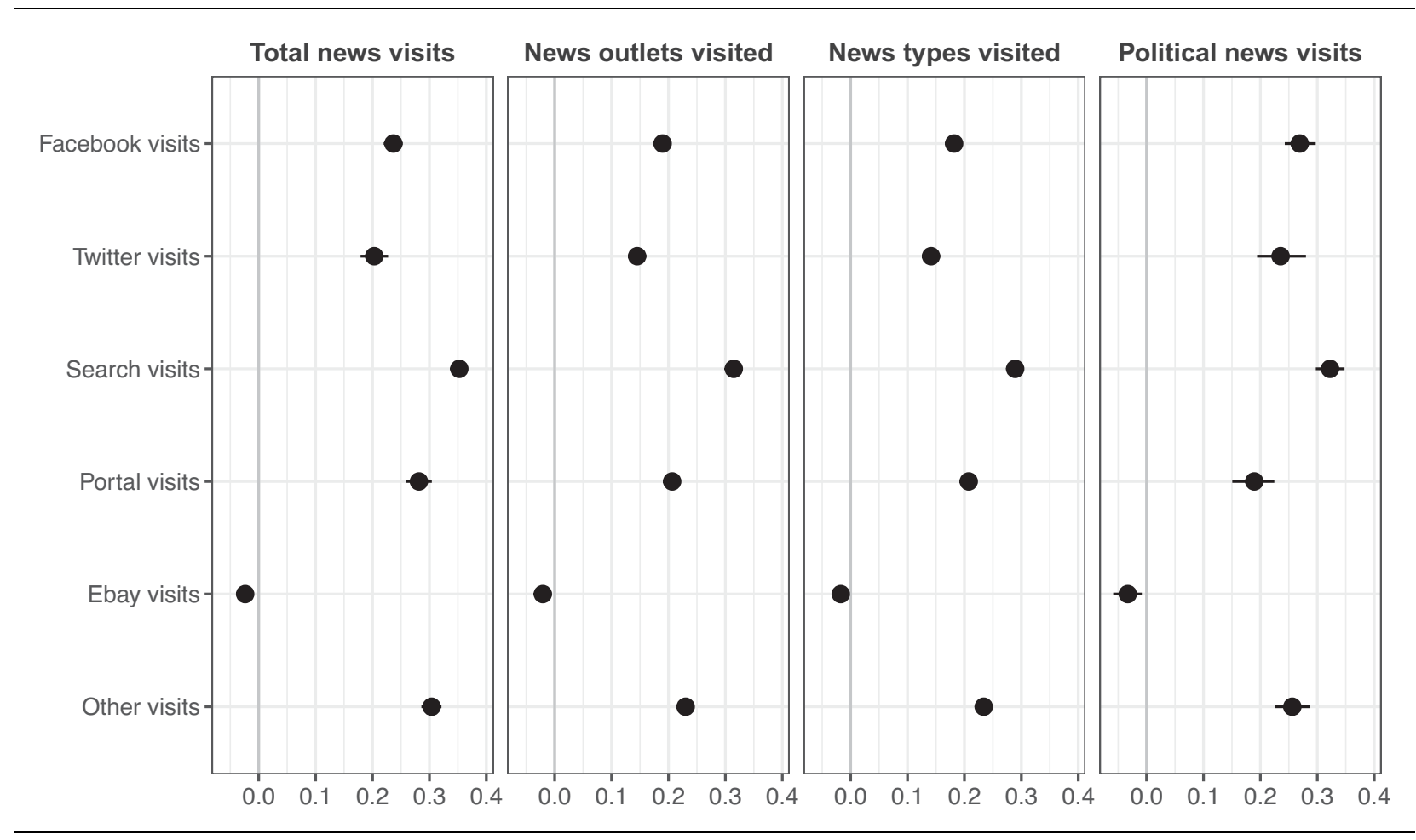

Note: Within-person Poisson regression coefficients and $99 \%$ confidence intervals from REWB models. $N=7,754$ persons; 486,789 person-days. Figure S8 displays the estimated variability of the effects.

To provide a more nuanced view on news exposure and increase the identification power, we conducted a longitudinal analysis of the variability in participants' web browsing behavior across the three-month research period. For this, we aggregated visits on the daily level and used the random-effects within-between (REWB) model (Bell, Fairbrother, and Jones 2019) to separate the effects of regular online habits (betweenperson differences) from the effects of daily fluctuations in the use of intermediaries on nonregular news exposure (within-person effects), controlling for overall daily online activity (Other visits) and using a Poisson estimation to account for the dependent count variables (see SM S7 for technical details). In order to capture possible effect heterogeneity, all within-person effects were allowed to vary across participants (person-level random slopes).

We constructed two dependent variables, similar to previous studies (Fletcher and Nielsen 2018a; Scharkow et al. 2020): the total number of news visits and the number of visited news outlets. Figure 2 shows that using more intermediaries on a given day positively predicts both outcomes. For instance, a person who was using search engines twice as often than on a typical day is estimated to consume $28 \%$ more news articles published by $24 \%$ more news outlets. The effects were positively significant for all types of intermediaries and general online activity, yet negative for the placebo eBay. Moreover, the estimated slopes were positive for almost all participants and all intermediaries
(SM Figure S8). In line with cross-sectional survey research (Fletcher and Nielsen 2018a), between-person effects were also consistently positive (SM Figure S9).

The model results for two additional dependent variables speak against concerns about a shrinking diversity of news sources and avoidance of political news in digital high-choice media environments: Figure 2 demonstrates that higher usage of any type of intermediary on a given day was also positively related to more diverse news types among the daily news visits and more political news exposure. ${ }^{3}$

Although the fixed effects reported in Figure 2 establish that social media, search, and portal visits foster news exposure on average, there could be crosscountry and person-level differences such that not all individuals profit equally from using intermediaries. We therefore investigated the moderating effects of country, age, gender, education, political interest, and political extremism. ${ }^{4}$ The corresponding results are reported in Figure 3. Turning to macro-level differences first, we see some cross-national variation. Most

\footnotetext{
${ }^{3}$ The correlations between the dependent variables were highest for the number of news sources and unique news types (Kendall's $\tau=0.84)$. For the other pairs, correlations ranged from $\tau=0.29$ to $\tau=0.53$, indicating that these measures tapped into related, yet different behaviors.

${ }^{4}$ The direct effects of each covariate on news exposure were in line with existing research: older, male, highly educated, and politically interested citizens consumed more news (SM Figure S10).
} 
FIGURE 3. Variability of Within-Person Effects across Countries and Personal Characteristics
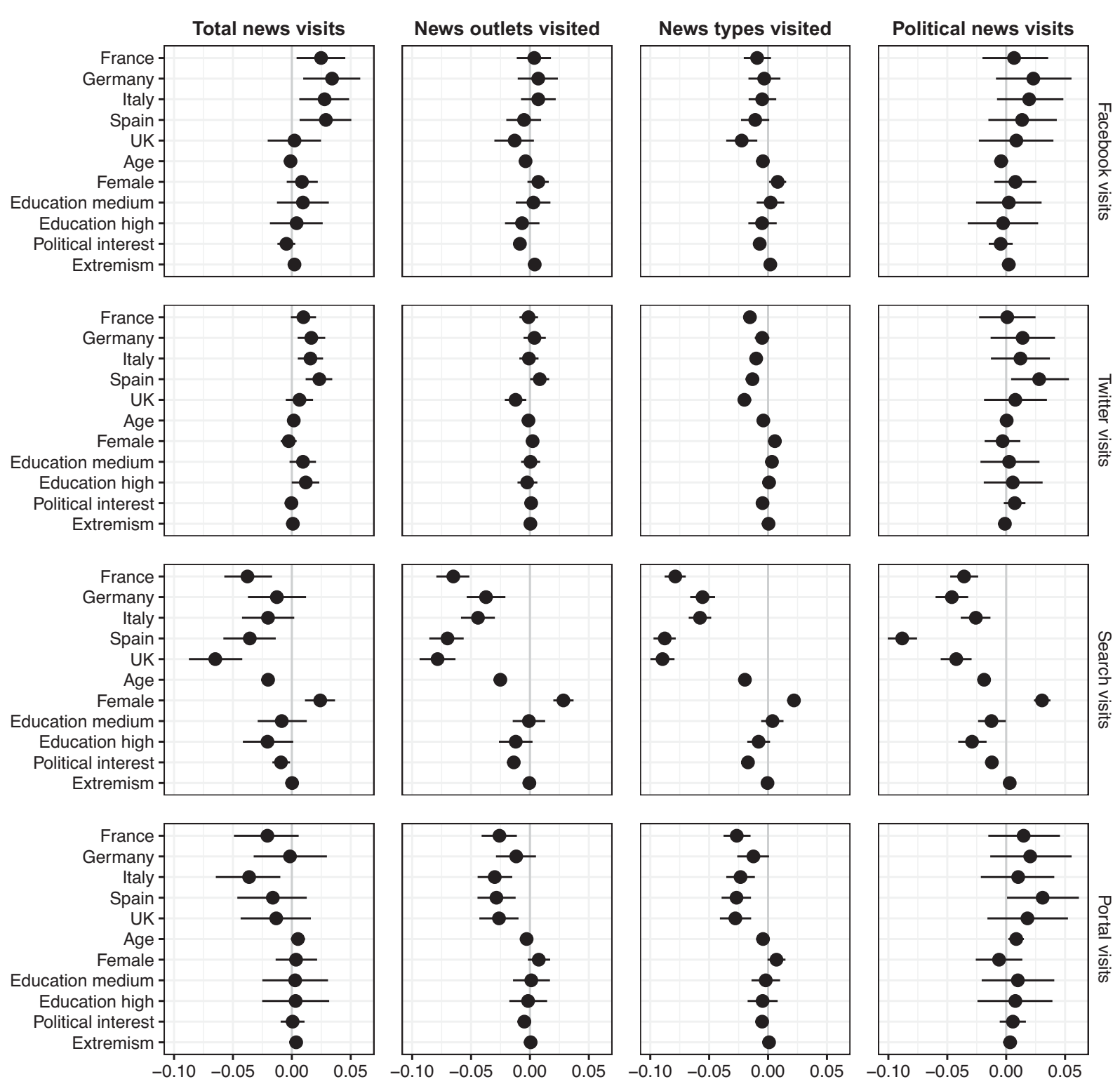

Note: Regression coefficients and 99\% confidence intervals from moderation analyses of the random within-person slopes of the REWB model. Coefficients describe how, for any level of the moderating variable, the within-person effects of using intermediaries on news exposure deviated from the fixed effects displayed in Figure 2. Reference categories are "US" and "Education low." Age was divided by 10 before the estimation to improve interpretation. $N=7,622$ persons; 478,647 person-days.

consistently, intermediaries had stronger effects on the number of news outlets and news types in the US (the reference country), especially when it comes to using search engines and portals. On the individual level, deviations from the fixed effects were most pronounced for search engines, which increased news exposure more strongly for less politically interested, younger, and female online users. Notwithstanding some statistically significant effects, the most important finding from Figure 3 is the overarching homogeneity of the within-person intermediary effects across all personal characteristics and countries. Even the most pronounced deviations from the average effects in Figure 2 were so small that the resulting conditional effects were still positive and significantly different from zero in all subgroups. For example, even the seemingly strong positive deviation for females when using search engines twice as often than on a regular day merely translates into $30 \%$ more news visits per day compared with the average effect of $28 \%$.

With its cross-country design, the paper goes beyond previous web tracking studies that have investigated polarization in the US online information environment in isolation (Bakshy, Messing, and Adamic 2015; 
Flaxman, Goel, and Rao 2016; Guess 2021). We conducted an additional analysis to establish comparability with these studies and test whether using intermediaries biases news exposure in line with political predispositions. In models for US study participants relying on domain ideology data by Bakshy, Messing, and Adamic (2015), media diet slant was not reinforced through intermediaries, according to neither party identification nor ideological self-placement (SM S8.4). Finally, robustness tests including selfreported measures of active news engagement, political discussion behavior, and social network characteristics (Lee and Kim 2017; Thorson 2020; Figure S11) as well as using data from smartphones that were available for $36 \%$ of study participants (SM S8.3) confirmed the main results.

\section{CONCLUSION}

Online intermediaries such as Facebook or Google are often accused of contributing to the perils of democracy by exacerbating (political) news avoidance and onesided content exposure. Yet our large-scale observation of web browsing behavior showed that intermediaries foster exposure to political and nonpolitical news and its breadth in terms of the news sources and news types used. These effects were broadly consistent across types of intermediaries (social network sites, search engines, portals) and diverse political and media systems, and they were not strongly moderated by individual-level differences. Besides directly moderating media diets ideologically (Barberá 2015; Guess 2021), online intermediaries indirectly expose users to news content they would otherwise not see or click on. As such, the big online platforms counteract the fragmentation tendencies of the "post-broadcast democracy" that has been characterized by a proliferation of niche content (Prior 2007).

While individual-level web browsing histories coupled with surveys provide an unprecedented granularity, more superficial news exposure within platforms themselves is still not captured by tracking tools. Reassuringly, first academic evidence relying on data from the Facebook News Feed shows that the bulk of news exposure concentrates on URLs from credible news sources (Guess et al. 2021), suggesting that the platform creates opportunity structures for "incidental" and more substantive encounters with public-affairs coverage. Combining such insights with repeated in situ surveys would greatly enhance our capability to explore users' varied motivations and reactions to contents while navigating the web. Importantly, more research should be devoted to the downstream political effects of socially mediated information exposure (Carlson 2019).

Some widely held assumptions about the negative consequences of intermediaries did not withstand scrutiny in our analysis, as there were no indications that intermediaries exacerbate inequalities in news exposure. At the same time, the evidence yielded only weak support that otherwise less avid news users benefit relatively more from intermediary use. The generally limited effect heterogeneity we identified corresponds with recent research showing that most online users devote only a marginal fraction of their online activities to news and that ideology has a minor influence (Fletcher, Robertson, and Nielsen 2021; Guess 2021; Scharkow et al. 2020). Because concerns about apathetic citizens remain critical in an increasingly digital media environment (Prior 2007; Van Aelst et al. 2017), it is hard to overstate intermediaries' role in establishing a minimum level of exposure to news and political information. And yet Facebook's changes to its news feed that attempt to prioritize the content of friends at the expense of (news) organizations still illustrated that the big online platforms can change algorithms at their own discretion, with potentially profound democratic consequences. ${ }^{5}$

\section{SUPPLEMENTARY MATERIALS}

To view supplementary material for this article, please visit http://doi.org/10.1017/S0003055421001222.

\section{DATA AVAILABILITY STATEMENT}

Research documentation and data that support the findings of this study are openly available at the American Political Science Review Dataverse: https://doi.org/ 10.7910/DVN/P3XVFO. Limitations on data availability are discussed in the text.

\section{ACKNOWLEDGMENTS}

We thank three reviewers, the editors, and panel participants at the ECPR General Conference 2020 for helpful comments. We are grateful to Caterina Froio, Justin Ho, Nora Kirkizh, Ralph Schroeder, and Pu Yan for their contributions to the data collection and the coding of the news articles. We thank Ina Böckmann and Céline Widera for their excellent research assistance.

\section{FUNDING STATEMENT}

Sebastian Stier is grateful to the Volkswagen Foundation for funding the data collection (grant number $94758)$.

\section{CONFLICT OF INTEREST}

The authors declare no ethical issues or conflicts of interest in this research.

\footnotetext{
$\overline{5}$ https://www.nytimes.com/2018/01/11/technology/facebook-newsfeed.html.
} 


\section{ETHICAL STANDARDS}

The authors declare that the data collection involving human subjects in this article was reviewed and approved by the Oxford Internet Institute's Departmental Research Ethics Committee at the University of Oxford. The certificate is provided on the Harvard Dataverse. The authors affirm that this article adheres to the APSA's Principles and Guidance on Human Subjects Research.

\section{REFERENCES}

Anspach, Nicolas M. 2017. "The New Personal Influence: How Our Facebook Friends Influence the News We Read." Political Communication 34 (4): 590-606.

Bakshy, Eytan, Solomon Messing, and Lada A. Adamic. 2015. "Exposure to Ideologically Diverse News and Opinion on Facebook." Science 348 (6239): 1130-32.

Barberá, Pablo. 2015. "How Social Media Reduces Mass Political Polarization: Evidence from Germany, Spain, and the U.S." Working Paper. http://pablobarbera.com/static/barbera polarization_APSA.pdf.

Bell, Andrew, Malcolm Fairbrother, and Kelvyn Jones. 2019. "Fixed and Random Effects Models: Making an Informed Choice." Quality \& Quantity 53 (2): 1051-74.

Bode, Leticia. 2016. "Pruning the News Feed: Unfriending and Unfollowing Political Content on Social Media." Research \& Politics 3 (3). https://doi.org/10.1177/2053168016661873.

Carlson, Taylor N. 2019. "Through the Grapevine: Informational Consequences of Interpersonal Political Communication." American Political Science Review 113 (2): 325-39.

Flaxman, Seth, Sharad Goel, and Justin M. Rao. 2016. "Filter Bubbles, Echo Chambers, and Online News Consumption." Public Opinion Quarterly 80 (S1): 298-320.

Fletcher, Richard, and Rasmus Kleis Nielsen. 2018a. “Are People Incidentally Exposed to News on Social Media? A Comparative Analysis." New Media \& Society 20 (7): 2450-68.

Fletcher, Richard, and Rasmus Kleis Nielsen. 2018b. "Automated Serendipity: The Effect of Using Search Engines on News Repertoire Balance and Diversity." Digital Journalism 6 (8): 976-89.

Fletcher, Richard, Craig T. Robertson, and Rasmus Kleis Nielsen. 2021. "How Many People Live in Politically Partisan Online News Echo Chambers in Different Countries?" Journal of Quantitative Description: Digital Media 1. https://doi.org/10.51685/jqd.2021.020.

Guess, Andrew M. 2021. "(Almost) Everything in Moderation: New Evidence on Americans' Online Media Diets.” American Journal of Political Science. https://doi.org/10.1111/ajps.12589.

Guess, Andrew M., Kevin Aslett, Joshua Tucker, Richard Bonneau, and Jonathan Nagler. 2021. "Cracking Open the News Feed: Exploring What U.S. Facebook Users See and Share with LargeScale Platform Data." Journal of Quantitative Description: Digital Media 1. https://doi.org/10.51685/jqd.2021.006.
Hallin, Daniel C., and Paolo Mancini. 2004. Comparing Media Systems: Three Models of Media and Politics. Cambridge: Cambridge University Press.

Kalogeropoulos, Antonis, Richard Fletcher, and Rasmus Kleis Nielsen. 2019. "News Brand Attribution in Distributed Environments: Do People Know Where They Get Their News?" New Media \& Society 21 (3): 583-601.

Kümpel, Anna S. 2020. "The Matthew Effect in Social Media News Use: Assessing Inequalities in News Exposure and News Engagement on Social Network Sites (SNS)." Journalism 21 (8): 1083-98.

Lee, Jae Kook, and Eunyi Kim. 2017. "Incidental Exposure to News: Predictors in the Social Media Setting and Effects on Information Gain Online." Computers in Human Behavior 75: 1008-15.

Möller, Judith, Robbert N. van de Velde, Lisa Merten, and Cornelius Puschmann. 2020. "Explaining Online News Engagement Based on Browsing Behavior: Creatures of Habit?" Social Science Computer Review 38 (5): 616-32.

Prior, Markus. 2007. Post-Broadcast Democracy: How Media Choice Increases Inequality in Political Involvement and Polarizes Elections. Cambridge: Cambridge University Press.

Prior, Markus. 2009. "The Immensely Inflated News Audience: Assessing Bias in Self-Reported News Exposure." Public Opinion Quarterly 73 (1): 130-43.

Scharkow, Michael, Frank Mangold, Sebastian Stier, and Johannes Breuer. 2020. "How Social Network Sites and Other Online Intermediaries Increase Exposure to News." Proceedings of the National Academy of Sciences 117 (6): 2761-63.

Skovsgaard, Morten, and Kim Andersen. 2020. "Conceptualizing News Avoidance: Towards a Shared Understanding of Different Causes and Potential Solutions." Journalism Studies 21 (4): 459-76.

Stier, Sebastian, Frank Mangold, Michael Scharkow, and Johannes Breuer. 2021. "Replication Data for: Post Post-Broadcast Democracy? News Exposure in the Age of Online Intermediaries." Harvard Dataverse. Dataset. https://doi.org/ 10.7910/DVN/P3XVFO.

Stier, Sebastian, Johannes Breuer, Pascal Siegers, and Kjerstin Thorson. 2020. "Integrating Survey Data and Digital Trace Data: Key Issues in Developing an Emerging Field.” Social Science Computer Review 38 (5): 503-16.

Sunstein, Cass R. 2009. Republic.com 2.0. Princeton, NJ: Princeton University Press.

Thorson, Kjerstin. 2020. "Attracting the News: Algorithms, Platforms, and Reframing Incidental Exposure.” Journalism 21 (8): 1067-82.

Tucker, Joshua A., Andrew M. Guess, Pablo Barberá, Cristian Vaccari, Alexandra Siegel, Sergey Sanovich, Denis Stukal, and Brendan Nyhan. 2018. "Social Media, Political Polarization, and Political Disinformation: A Review of the Scientific Literature." Report. https://www.hewlett.org/wpcontent/uploads/2018/03/Social-Media-Political-Polarization-andPolitical-Disinformation-Literature-Review.pdf.

Van Aelst, Peter, Jesper Strömbäck, Toril Aalberg, Frank Esser, Claes de Vreese, Jörg Matthes, David Hopmann, et al. 2017. "Political Communication in a High-Choice Media Environment: A Challenge for Democracy?" Annals of the International Communication Association 41 (1): 3-27. 